\title{
Ionization for Three Dimensional Time-dependent Point Interactions
}

\author{
Michele Correggi* \\ International School for Advanced Studies SISSA/ISAS, Trieste, Italy \\ Gianfausto Dell'Antonio ${ }^{\dagger}$ \\ Centro Linceo Interdisciplinare ${ }^{\ddagger}$ Roma, Italy \\ Rodolfo Figari ${ }^{\S}$ \\ Dipartimento di Scienze Fisiche, Università di Napoli "Federico II" \\ and Sezione INFN, Napoli, Italy \\ Andrea Mantile \\ Dipartimento di Matematica e Applicazioni \\ Università di Napoli "Federico II", Napoli, Italy
}

\begin{abstract}
We study the time evolution of a three dimensional quantum particle under the action of a time-dependent point interaction fixed at the origin. We assume that the "strength" of the interaction $\alpha(t)$ is a periodic function with an arbitrary mean. Under very weak conditions on the Fourier coefficients of $\alpha(t)$, we prove that there is complete ionization as $t \rightarrow \infty$, starting from a bound state at time $t=0$. Moreover we prove also that, under the same conditions, all the states of the system are scattering states.
\end{abstract}

Ref. SISSA/ISAS preprint 11/2004/FM

\section{Introduction}

We shall study the time evolution of a three dimensional system with timedependent Hamiltonian given by

$$
H(t)=H_{0}+H_{I}(t)
$$

\footnotetext{
*E-mail address: correggi@sissa.it

$\dagger$ E-mail address: gianfa@sissa.it

‡On leave from Dipartimento di Matematica, Università di Roma, "La Sapienza", Italy.

$\S$ E-mail address: figari@na.infn.it

IE-mail address: andrea.mantile@dma.unina.it
} 
where the "perturbation" $H_{I}(t)$ is a zero-range interaction with time-dependent (periodic) "strength". In particular we are interested in proving complete ionization of the system as $t \rightarrow \infty$, starting from an initial condition at $t=0$ given by a bound state of the system. By complete ionization one can mean two different statements. The weaker one is that the survival probability of the bound state, i.e. the square modulus of the scalar product of the state at time $t$ with the bound state, goes to zero as $t \rightarrow \infty$. The stronger one is that every state $\Psi$ in the Hilbert space of the system is a scattering state (see for example 11, 14]) of $H(t)$, i.e. for every compact set $S \subset \mathbb{R}^{3}$,

$$
\lim _{t \rightarrow \infty} \frac{1}{t} \int_{0}^{t} d \tau \int_{S} d^{3} \vec{x}\left|\Psi_{\tau}(\vec{x})\right|^{2}=0
$$

$\Psi_{t}$ denoting the time evolution of the state $\Psi$. The last statement is related to the absence of eigenvalues of the Floquet operator associated to $H(t)$ (see 15, 13, 20]).

The usual way to deal with problems of this kind is by means of time-dependent perturbation theory and Fermi's golden rule, which gives for the survival probability the well known exponential decay for each order $n$ in the perturbative expansion. On the other hand simple examples of regular perturbations show that the survival probability decays to zero as a power-law (i.e. the limits $t \rightarrow \infty$ and $n \rightarrow \infty$ can not be interchanged). When the perturbation is not small, it is in general very difficult to solve the problem and find the law of decay. Therefore it is interesting to find models in which a non-perturbative solution exists and study the survival probability. In this paper we study one such model, in which $H_{I}(t)$ is given by a three dimensional point interaction. We shall see that it is possible to prove asymptotic complete ionization and find a power law decay for the survival probability, under generic condition on the scattering length ${ }^{1}$.

The one-dimensional version of the same problem has been widely analyzed in [4. [5] 6] 7], where complete ionization is proved under a suitable and very weak condition on the Fourier coefficients of the strength of the interaction. We shall see that the same genericity condition is also sufficient in three dimensions to have complete ionization of the system.

From a physical point of view, the model we are going to study is related to the strong laser ionization of Rydberg atoms ${ }^{2}$, showing many features of experimental data. Indeed, despite of the simplicity of the model, as in the one-dimensional case, it is possible to reproduce many effects of multiphoton ionization of excited hydrogen atoms by microwave field, with a good agreement with experiments (see 8]).

\section{The model}

The model we are going to study is a quantum particle subjected to a timedependent point interaction fixed at the origin in three dimensions, namely a system defined by the time-dependent self-adjoint Hamiltonian $H_{\alpha(t)}$,

$$
\mathcal{D}\left(H_{\alpha(t)}\right)=\left\{\Psi \in L^{2}\left(\mathbb{R}^{3}\right) \mid \exists q_{\lambda}(t) \in \mathbb{C},\left(\Psi(\vec{x})-q_{\lambda}(t) \mathcal{G}^{\lambda}(\vec{x})\right) \in H^{2}\left(\mathbb{R}^{3}\right),\right.
$$

\footnotetext{
${ }^{1}$ In three dimensions the parameter $\alpha(t)$ is proportional to the inverse of the scattering length.

${ }^{2}$ See the discussion contained in 40 and references therein.
} 


$$
\begin{gathered}
\left.\left.\left(\Psi-q_{\lambda}(t) \mathcal{G}^{\lambda}\right)\right|_{\vec{x}=0}=\left(\alpha(t)+\frac{\sqrt{\lambda}}{4 \pi}\right) q_{\lambda}(t)\right\} \\
\left(H_{\alpha(t)}+\lambda\right) \Psi=\left(H_{0}+\lambda\right)\left(\Psi-q_{\lambda}(t) \mathcal{G}^{\lambda}\right)
\end{gathered}
$$

where $\lambda \in \mathbb{R}, \lambda>0$ and

$$
\mathcal{G}^{\lambda}\left(\vec{x}-\vec{x}^{\prime}\right)=\frac{e^{-\sqrt{\lambda}\left|\vec{x}-\vec{x}^{\prime}\right|}}{4 \pi\left|\vec{x}-\vec{x}^{\prime}\right|}
$$

is the Green function of the free Hamiltonian $H_{0}=-\Delta$.

The operator ${ }^{3}(2.2)$ has absolutely continuous spectrum if $\alpha(t)$ is positive, while, when $\alpha(t)<0$, there exists exactly one negative eigenvalue $-(4 \pi \alpha(t))^{2}$, with normalized eigenfunction

$$
\varphi_{\alpha(t)}(\vec{x}) \equiv \frac{\sqrt{2|\alpha(t)|} e^{4 \pi \alpha(t)|\vec{x}|}}{|\vec{x}|}
$$

It is well known (see 9, 10, 12, 18, 19]) that the operator 22.2) defines a time propagation $U(t, s)$ given by a two-parameters unitary family, solving the timedependent Shrödinger equation

$$
i \frac{\partial \Psi_{t}}{\partial t}=H_{\alpha(t)} \Psi_{t}
$$

and

$$
\Psi_{t}(\vec{x})=U(t, s) \Psi_{s}(\vec{x})=U_{0}(t-s) \Psi_{s}(\vec{x})+i \int_{s}^{t} d \tau q(\tau) U_{0}(t-\tau ; \vec{x})
$$

where $U_{0}(t)=\exp \left(-i H_{0} t\right), U_{0}(t ; \vec{x})$ is the kernel associated to the free propagator and the charge $q(t)$ satisfies a Volterra integral equation for $t \geq s$,

$$
q(t)+4 \sqrt{\pi i} \int_{s}^{t} d \tau \frac{\alpha(\tau) q(\tau)}{\sqrt{t-\tau}}=4 \sqrt{\pi i} \int_{s}^{t} d \tau \frac{\left(U_{0}(\tau) \Psi_{s}\right)(0)}{\sqrt{t-\tau}}
$$

We are interested in studying complete ionization of system defined by (2.2) and (2.4), starting from initial conditions

$$
\Psi_{0}(\vec{x})=\varphi_{\alpha(0)}(\vec{x})
$$

$\varphi_{\alpha(0)}(\vec{x})$ being the bound state ${ }^{4}$ of $H_{\alpha(0)}$.

We shall assume that $\alpha(t)$ is a real periodic continuous function with period $T$. The meaningful parameter of the system is the negative lower bound of $\alpha(t)$. Indeed, if $\inf (\alpha(t)) \geq 0$, the wave operator associated to $\left(H_{0}, H_{\alpha(t)}\right)$ is unitary (see [19]) so that any initial state evolves into a scattering state (see also the remark at the end of section 5). Hence we require that

$$
\text { 1. } \alpha(0)<0
$$

\footnotetext{
${ }^{3}$ For a general review about point interactions see 1$] 2$ and references therein.

${ }^{4}$ In order to do this analysis we shall require that $\alpha(0)<0$.
} 
Continuity of $\alpha(t)$ guarantees that it can be decomposed in a Fourier series, for each $t \in \mathbb{R}^{+}$, and the series converges uniformly on every compact subset of the real line. In terms of the Fourier coefficients of $\alpha(t)$, we assume

$$
\begin{aligned}
& \text { 2. } \alpha(t)=\sum_{n \in \mathbb{Z}} \alpha_{n} e^{-i n \omega t},\left\{\alpha_{n}\right\} \in \ell_{1}(\mathbb{Z}), \omega=\frac{2 \pi}{T} \\
& \text { 3. } \alpha_{n}=\alpha_{-n}^{*}
\end{aligned}
$$

We start noticing that from (2.6) we have

$$
|q(t)| \leq 4 \sqrt{\pi} \sup (|\alpha|) \int_{0}^{t} d \tau \frac{|q(\tau)|}{\sqrt{t-\tau}}+4 \sqrt{\pi} \int_{0}^{t} d \tau \frac{\left|\left(U_{0}(\tau) \Psi_{0}\right)\right|(0)}{\sqrt{t-\tau}}
$$

from which we deduce that $\eta(t)-|q(t)| \geq 0$, if $\eta(t)$ is the unique solution of the equation

$$
\eta(t)=4 \sqrt{\pi} \sup (|\alpha|) \int_{0}^{t} d \tau \frac{\eta(\tau)}{\sqrt{t-\tau}}+4 \sqrt{\pi} \int_{0}^{t} d \tau \frac{\left|\left(U_{0}(\tau) \Psi_{0}\right)\right|(0)}{\sqrt{t-\tau}}
$$

Iterating (2.11) once and differentiating we obtain for $\eta$ the differential equation

$$
\frac{d \eta}{d t}=16 \pi^{2}(\sup (|\alpha|))^{2} \eta+16 \pi^{2}\left|\left(U_{0}(t) \Psi_{0}\right)\right|(0)
$$

where the inhomogeneous term is finite at each time $t$ with, at most, an integrable singularity at $t=0$. We conclude that

$$
|q(t)| \leq \eta(t) \leq C e^{16 \pi^{2}(\sup (|\alpha|))^{2} t}
$$

As a consequence the Laplace transform of $q(t)$, denoted by

$$
\tilde{q}(p) \equiv \int_{0}^{\infty} d t e^{-p t} q(t)
$$

exists analytic at least for $\Re(p)>16 \pi^{2}(\sup (|\alpha|))^{2}$.

Applying the Laplace transform to equation (2.6), one has

$$
\tilde{q}(p)=-4 \pi \sqrt{\frac{i}{p}} \sum_{k \in \mathbb{Z}} \alpha_{k} \tilde{q}(p+i \omega k)+\tilde{f}(p)
$$

where

$$
\begin{gathered}
\tilde{f}(p) \equiv \frac{2 \sqrt{2|\alpha(0)|}}{\pi} \sqrt{\frac{i}{p}} \int_{0}^{\infty} d t e^{-p t} \int_{\mathbb{R}^{3}} d^{3} \vec{k} \frac{e^{-i k^{2} t}}{k^{2}+(4 \pi \alpha(0))^{2}}= \\
=8 \sqrt{\frac{2|\alpha(0)|}{i p}} \int_{0}^{\infty} d k \frac{k^{2}}{\left(k^{2}+(4 \pi \alpha(0))^{2}\right)\left(k^{2}-i p\right)}= \\
=4 \pi i \sqrt{\frac{2|\alpha(0)|}{-i p}} \frac{4 \pi \alpha(0)+\sqrt{-i p}}{(4 \pi \alpha(0))^{2}+i p}
\end{gathered}
$$


and with the choice of the branch cut for the square root along the negative real line: if $p=\varrho e^{i \vartheta}$,

$$
\sqrt{p}=\sqrt{\varrho} e^{i \vartheta / 2}
$$

with $-\pi<\vartheta \leq \pi$.

By unitarity of the evolution (2.4), it follows that the Laplace transform of $q(t)$ is indeed analytic on the open right half plane:

Proposition 2.1 The Laplace transform of $q(t)$, solution of (2.6), is analytic at least for $\Re(p)>0$.

Proof: Using the decomposition of the wave function at time $t$ defined by (2.5), we can write the survival probability in the following way:

$$
\begin{gathered}
\theta(t) \equiv\left(\varphi_{\alpha(0)}, \Psi_{t}\right)_{L^{2}\left(\mathbb{R}^{3}\right)}=\left(\varphi_{\alpha(0)}, e^{-i H_{0} t} \varphi_{\alpha(0)}\right)_{L^{2}\left(\mathbb{R}^{3}\right)}+ \\
+i\left(\varphi_{\alpha(0)}(\vec{x}), \int_{0}^{t} d \tau q(\tau) U_{0}(t-\tau ; \vec{x})\right)_{L^{2}\left(\mathbb{R}^{3}\right)}
\end{gathered}
$$

Let us define

$$
Z_{1}(t) \equiv\left(\varphi_{\alpha(0)}, e^{-i H_{0} t} \varphi_{\alpha(0)}\right)_{L^{2}\left(\mathbb{R}^{3}\right)}
$$

By the usual dissipative estimate for the free propagator, one has

$$
\left|Z_{1}(t)\right| \leq c_{1} t^{-\frac{3}{2}}
$$

as $t \rightarrow \infty$ for some constant $c_{1} \in \mathbb{R}$. Hence $Z_{1}(t)$ belongs to $L^{1}\left(\mathbb{R}^{+}\right)$and then its Laplace transform $\tilde{Z}_{1}(p)$ is analytic at least for $\Re(p) \geq 0$.

The second piece of the scalar product is given by

$$
\begin{gathered}
Z(t) \equiv i\left(\varphi_{\alpha(0)}(\vec{x}), \int_{0}^{t} d \tau q(\tau) U_{0}(t-\tau ; \vec{x})\right)_{L^{2}\left(\mathbb{R}^{3}\right)}= \\
\quad=i \int_{0}^{t} d \tau q(\tau)\left(e^{-i H_{0}(t-\tau)} \varphi_{\alpha(0)}\right)(0)
\end{gathered}
$$

and taking the Laplace transform of $Z(t)$, we have

$$
\tilde{Z}(p)=\tilde{Z}_{2}(p) \tilde{q}(p)
$$

where

$$
\tilde{Z}_{2}(p) \equiv-\frac{4 \sqrt{2 \pi|\alpha(0)|}}{4 \pi \alpha(0)-\sqrt{-i p}}
$$

is analytic for $\Re(p)>0$ and never equal to 0 , because of condition (2.8).

Hence the Laplace transform of $\theta(t)$ is given by

$$
\tilde{\theta}(p)=\tilde{Z}_{1}(p)+\tilde{Z}_{2}(p) \tilde{q}(p)
$$

But $\theta(t)$ is a bounded function ${ }^{5}$, because of unitarity of the evolution (2.4), and then its Laplace transform is analytic on the open right half plane. The claim then follows from analyticity of $\tilde{Z}_{1}(p), \tilde{Z}_{2}(p)$ and absence of zeros of $\tilde{Z}_{2}(p)$.

\footnotetext{
${ }^{5}$ Actually $|\theta(t)| \leq 1$, since the initial state is normalized.
} 
A well known property of Volterra integral operators, with regular or weakly singular kernel, implies

Proposition 2.2 The homogeneous equations associated to (2.6) has no nonzero solution in $L_{\mathrm{loc}}^{p}\left(\mathbb{R}^{+}\right), 1 \leq p \leq \infty$.

Proof: The proof (see e.g. [16]) exploits the fact that the $n$-fold iterated kernel is a contraction in any $L^{p}\left(0, T_{n}\right)$ with $T_{n}$ increasing to infinity for increasing $n$.

In the following sections we shall prove asymptotic complete ionization of the system under generic conditions on $\alpha(t)$. Although the result does not depend on the sign of the mean $\alpha_{0}$ of $\alpha(t)$, we have to discuss separately the case $\alpha_{0}<0$ and $\alpha_{0} \geq 0$, because of the slightly different form of equation (2.14).

\section{CASE I: $\alpha_{0}<0$}

Since $\alpha(0)<0$, changing the energy scale, it is always possible to assume that $\alpha(t)$ satisfies the normalization

$$
\text { 4. } \alpha(0)=\sum_{n \in \mathbb{Z}} \alpha_{n}=-\frac{1}{4 \pi}
$$

Moreover we introduce another condition we shall use later on: let $\mathcal{T}$ the right shift operator on $\ell_{1}(\mathbb{N})$, i.e.

$$
(\mathcal{T} a)_{n} \equiv a_{n+1}
$$

we say that $\alpha=\left\{\alpha_{n}\right\} \in \ell_{1}(\mathbb{Z})$ is generic with respect to $\mathcal{T}$, if $\tilde{\alpha} \equiv\left\{\alpha_{n}\right\}_{n>0} \in$ $\ell_{1}(\mathbb{N})$ satisfies the following condition

$$
e_{1}=(1,0,0, \ldots) \in \overline{\bigvee_{n=0}^{\infty} \mathcal{T}^{n} \tilde{\alpha}}
$$

For a detailed discussion of genericity condition see 4 .

If (3.1) holds, equation (2.14) becomes (at least for $\Re(p)>0$ )

$$
\tilde{q}(p)=-\frac{4 \pi}{4 \pi \alpha_{0}+\sqrt{-i p}} \sum_{\substack{k \in \mathbb{Z} \\ k \neq 0}} \alpha_{k} \tilde{q}(p+i \omega k)-\frac{2 i \sqrt{2 \pi}}{4 \pi \alpha_{0}+\sqrt{-i p}} \frac{1-\sqrt{-i p}}{1+i p}
$$

and by Proposition 2.1] its solution is analytic on the open right half plane. In the following section we shall extend the equation (3.4) above to the imaginary axis and study the behavior of the solution there. 


\subsection{Behavior on the imaginary axis at $p \neq 0$}

Setting $q_{n}(p) \equiv \tilde{q}(p+i \omega n)$, we obtain a sequence of functions on the strip $\mathcal{I}=\{p \in \mathbb{C}, 0 \leq \Im(p)<\omega\}$. Setting

$$
q(p) \equiv\left\{q_{n}(p)\right\}_{n \in \mathbb{Z}}
$$

equation (3.4) can be rewritten

$$
q(p)=\mathcal{L}(p) q(p)+g(p)
$$

where

$$
(\mathcal{L} q)_{n}(p) \equiv-\frac{4 \pi}{4 \pi \alpha_{0}+\sqrt{\omega n-i p}} \sum_{\substack{k \in \mathbb{Z} \\ k \neq 0}} \alpha_{k} q_{n+k}(p)
$$

and $g(p)=\left\{g_{n}(p)\right\}_{n \in \mathbb{Z}}$ with

$$
g_{n}(p) \equiv-\frac{2 i \sqrt{2 \pi}}{4 \pi \alpha_{0}+\sqrt{\omega n-i p}} \frac{1-\sqrt{\omega n-i p}}{1+i p-\omega n}
$$

From the explicit expression of the operator (3.6) and (3.7), it is clear that the coefficients of the equation fails to be analytic on the imaginary axis at $\bar{p}=\left(\left(4 \pi \alpha_{0}\right)^{2}-\omega \bar{n}\right) i$, for some $\bar{n} \in \mathbb{Z}$ and then the solution may be singular there.

Since $\Im(p) \in[0, \omega)$, one has

$$
\frac{\left(4 \pi \alpha_{0}\right)^{2}}{\omega}-1<\bar{n} \leq \frac{\left(4 \pi \alpha_{0}\right)^{2}}{\omega}
$$

and then the singularity appears at most in the equation for $q_{\bar{n}}$ (there is only one integer ${ }^{6}$ which satisfies the previous inequality) at $\bar{p}=\left(\left(4 \pi \alpha_{0}\right)^{2}-\omega \bar{n}\right) i$. For instance, if $\omega>\left(4 \pi \alpha_{0}\right)^{2}$, the pole may be at $\bar{p}=\left(4 \pi \alpha_{0}\right)^{2} i$ in the equation for $q_{0}$.

Actually we have to distinguish the so called (see 4]) resonant case, i.e. when

$$
\left(4 \pi \alpha_{0}\right)^{2}=N \omega
$$

for some $N \in \mathbb{N}$, because in that case we can have a pole only at $p=0$ and then the solution is immediately seen to be analytic on the whole imaginary axis except at most for $p=0$.

Let us first consider the behavior of the solution on the imaginary axis for $p \neq 0, \bar{p}$. We are going to prove that the solution is in fact analytic there. We prove first an important property of the operator $\mathcal{L}$ :

Proposition 3.1 For $p \in \mathcal{I}, \Re(p)=0, p \neq 0, \bar{p}, \mathcal{L}(p)$ is an analytic operatorvalued function and $\mathcal{L}(p)$ is a compact operator on $\ell_{2}(\mathbb{Z})$.

Proof: Analyticity on the imaginary axis for $p \neq 0, \bar{p}$ easily follows from the explicit expression of the operator.

Moreover $\mathcal{L}(p)$ can be written

$$
\mathcal{L}(p)=b(p) \sum_{\substack{k \in \mathbb{Z} \\ k \neq 0}} \alpha_{k} \mathcal{T}^{n+k}
$$

\footnotetext{
${ }^{6}$ In fact $\bar{n}$ must be non negative.
} 
where $b(p)$ is the operator

$$
(b q)_{n}(p) \equiv b_{n}(p) q_{n}(p)=-\frac{4 \pi q_{n}(p)}{4 \pi \alpha_{0}+\sqrt{\omega n-i p}}
$$

and $\mathcal{T}$ is the right shift operator on $\ell_{2}(\mathbb{Z})$.

Since $\|\mathcal{T}\|=1$, the series converges strongly to a bounded operator. Moreover $b(p)$ is a compact operator on the imaginary axis for $p \neq 0, \bar{p}: b(p)$ is the norm limit of a sequence of finite rank operators, because $\lim _{n \rightarrow \infty} b_{n}(p)=0$. Hence the result follows for example from Theorem VI.12 and VI.13 of [17].

Proposition 3.2 There exists a unique solution $q_{n}(p) \in \ell_{2}(\mathbb{Z})$ of (3.5) and it is analytic on the imaginary axis for $p \neq 0, \bar{p}$.

Proof: The key point will be the application of the analytic Fredholm theorem to the operator $\mathcal{L}(p)$ (Theorem VI.14 of [17]), in order to prove that $(I-\mathcal{L}(p))^{-1}$ exists for $p \neq 0, \bar{p}$.

Since there is no non-zero solution in $L_{\mathrm{loc}}^{2}\left(\mathbb{R}^{+}\right)$of the homogeneous equation associated to (2.6) (see the Proposition 2.2), then the homogeneous equation associated to (3.5) has only the trivial solution in $\ell_{2}(\mathbb{Z})$. Moreover the operator $\mathcal{L}$ is compact and thus analytic Fredholm theorem applies. The result easily follows, because $g(p) \in \ell_{2}(\mathbb{Z})$ and each $g_{n}(p)$ is analytic for $p \neq 0, \bar{p}$.

We can now study the equation (3.5) in a neighborhood of $\bar{p}$ (if $\bar{p} \neq 0$ ). An important preliminary result is the following

Lemma 3.1 Let (2.9) and the genericity condition (3.3) be satisfied by $\left\{\alpha_{n}\right\}$. The system of equations

$$
r_{n}=-\frac{4 \pi}{4 \pi \alpha_{0}+\sqrt{\omega n-i p}}\left\{\sum_{\substack{k \in \mathbb{Z} \\ k \neq n, \bar{n}}} \alpha_{k-n} r_{k}+h_{n}(p)\right\}
$$

has a unique solution $\left\{r_{n}\right\} \in \ell_{2}(\mathbb{Z} \backslash\{\bar{n}\})$ in a pure imaginary neighborhood of $\bar{p}$, where $\bar{n} \in \mathbb{Z}$ and $\bar{p} \in \mathcal{I}, \Re(\bar{p})=0$, are defined by [3.8), for every $h_{n}(p)$ such that

$$
h_{n}^{\prime}(p) \equiv \frac{h_{n}(p)}{4 \pi \alpha_{0}+\sqrt{\omega n-i p}}
$$

belongs to $\ell_{2}(\mathbb{Z} \backslash\{\bar{n}\})$.

Moreover, if $h_{n}(p)$ is analytic in a neighborhood of $\bar{p}$, the solution is analytic in the same neighborhood.

Proof: Equation (3.9) is of the form

$$
r=\mathcal{L}^{\prime} r+h^{\prime}
$$

where $h^{\prime} \equiv\left\{h_{n}^{\prime}\right\}$ belongs to $\ell_{2}(\mathbb{Z} \backslash\{\bar{n}\})$ and $\mathcal{L}^{\prime}$ is a compact operator (see Proposition 3.1).

In order to apply analytic Fredholm theorem to the operator $\mathcal{L}^{\prime}$, we need to prove 
that there is no non-zero solution in a neighborhood of $\bar{p}$ of the homogeneous equation. Suppose that the contrary is true, so that $\left\{R_{n}\right\} \in \ell_{2}(\mathbb{Z} \backslash\{\bar{n}\})$ is a non-zero solution of

$$
R_{n}=-\frac{4 \pi}{4 \pi \alpha_{0}+\sqrt{\omega n-i p}} \sum_{\substack{k \in \mathbb{Z} \\ k \neq n, \bar{n}}} \alpha_{k-n} R_{n}
$$

Multiplying both sides of equation above by $R_{n}^{*}$ and summing over $n \in \mathbb{Z} \backslash\{\bar{n}\}$, one has

$$
\sum_{\substack{n \in \mathbb{Z} \\ n \neq \bar{n}}} \sqrt{\omega n-i p}\left|R_{n}\right|^{2}=-4 \pi \sum_{\substack{n, k \in \mathbb{Z} \\ n, k \neq \bar{n}}} R_{n}{ }^{*} \alpha_{k-n} R_{k}
$$

and, since the right hand side is real,

$$
\Im\left[\sum_{\substack{n \in \mathbb{Z} \\ n \neq \bar{n}}} \sqrt{\omega n-i p}\left|R_{n}\right|^{2}\right]=0
$$

for $p=i \lambda, 0<\lambda<\omega$, and then $R_{n}=0$ for $n<0$. Now suppose that $R \neq 0$ and let $n_{0} \in \mathbb{N}$ be such that $R_{n}=0, n<n_{0}$, and $R_{n_{0}} \neq 0$ (hence $n_{0} \geq 0$ ). Fixing $R_{\bar{n}}=0$, for each $n<n_{0}$ the homogeneous equation gives

$$
\sum_{k=n_{0}}^{\infty} \alpha_{k-n} R_{k}=0
$$

or, setting $k=n_{0}-1+k^{\prime}$, for $n \geq 0$,

$$
\sum_{k^{\prime}=1}^{\infty} \alpha_{k^{\prime}+n} R_{n_{0}-1+k^{\prime}}=0
$$

which implies (see (2.9)), for each $n \geq 0$,

$$
\left(R^{\prime}, \mathcal{T}^{n} \alpha\right)_{\ell_{2}(\mathbb{N})}=0
$$

where $R_{n}^{\prime}=R_{n_{0}-1+n}^{*}$ and $(\cdot, \cdot)$ stands for the standard scalar product on $\ell_{2}(\mathbb{N})$. If $\left\{\alpha_{n}\right\}$ satisfies the genericity condition (3.3), $R^{\prime}$ has to be orthogonal also to $e_{1}$ and then $R_{n_{0}}=0$, which is a contradiction. Therefore $R=0$.

The first part of the Lemma then follows from analyticity of $\mathcal{L}^{\prime}(p)$ and analytic Fredholm theorem. Moreover if $\left\{h_{n}(p)\right\}$ is analytic in a neighborhood of $\bar{p}$, analyticity of the solution is a straightforward consequence.

Proposition 3.3 If $\left\{\alpha_{n}\right\}$ satisfies (2.9) and the genericity condition with respect to $\mathcal{T}$ (3.3), the unique solution $\left\{q_{n}\right\} \in \ell_{2}(\mathbb{Z})$ of (3.5) in analytic on the imaginary axis except at most for $p=0$.

Proof: If $\left(4 \pi \alpha_{0}\right)^{2}=N \omega$ for some $N \in \mathbb{N}$ (resonant case) there is nothing to prove, since the coefficients of (3.5) fails to be analytic only at $p=0$. On the other hand, in the non resonant case, Proposition 3.2 guarantees analyticity 
on imaginary axis for $p \neq 0, \bar{p}$. Therefore it is sufficient to study the behavior of the solution in a neighborhood of $\bar{p}$, where the coefficients of 3.5 have a singularity. We are going to prove that in fact the solution is analytic at $\bar{p}$.

The strategy of the proof is to analyze separately the terms $q_{n}, n \neq \bar{n}, \bar{n}$ being defined in (3.8), and then prove that also $q_{\bar{n}}$ is analytic in a neighborhood of $\bar{p}$. By Lemma 3.1 there is a unique solution of the system

$$
t_{n}=-\frac{4 \pi}{4 \pi \alpha_{0}+\sqrt{\omega n-i p}} \sum_{\substack{k \in \mathbb{Z} \\ k \neq n, \bar{n}}} \alpha_{k-n} t_{k}-\frac{4 \pi \alpha_{\bar{n}-n}}{4 \pi \alpha_{0}+\sqrt{\omega n-i p}}
$$

Setting $q_{n}=r_{n}+t_{n} q_{\bar{n}}, n \neq \bar{n}$, on (3.5), one has

$$
\begin{gathered}
r_{n}+t_{n} q_{\bar{n}}=-\frac{4 \pi}{4 \pi \alpha_{0}+\sqrt{\omega n-i p}}\left\{\alpha_{\bar{n}-n} q_{\bar{n}}+\sum_{\substack{k \in \mathbb{Z} \\
k \neq n, \bar{n}}} \alpha_{k-n}\left(r_{k}+t_{k} q_{\bar{n}}\right)\right\}+ \\
-\frac{2 i \sqrt{2 \pi}}{4 \pi \alpha_{0}+\sqrt{\omega n-i p}} \frac{1-\sqrt{\omega n-i p}}{1+i p-\omega n}
\end{gathered}
$$

and therefore the equation for $\left\{r_{n}\right\}, n \neq \bar{n}$, becomes

$$
r_{n}=-\frac{4 \pi}{4 \pi \alpha_{0}+\sqrt{\omega n-i p}}\left\{\sum_{\substack{k \in \mathbb{Z} \\ k \neq 0,-n}} \alpha_{k} r_{n+k}+\frac{i}{\sqrt{2 \pi}} \frac{1-\sqrt{\omega n-i p}}{1+i p-\omega n}\right\}
$$

while $q_{\bar{n}}$ satisfies the equation

$$
q_{\bar{n}}=-\frac{4 \pi}{4 \pi \alpha_{0}+\sqrt{\omega \bar{n}-i p}}\left\{\sum_{\substack{k \in \mathbb{Z} \\ k \neq \bar{n}}} \alpha_{k-\bar{n}}\left(r_{k}+t_{k} q_{\bar{n}}\right)+\frac{i}{\sqrt{2 \pi}} \frac{1-\sqrt{\omega \bar{n}-i p}}{1+i p-\omega \bar{n}}\right\}
$$

or

$$
\left[4 \pi \alpha_{0}+\sqrt{\omega \bar{n}-i p}+4 \pi \sum_{\substack{k \in \mathbb{Z} \\ k \neq \bar{n}}} \alpha_{k-\bar{n}} t_{k}\right] q_{\bar{n}}=-4 \pi \sum_{\substack{k \in \mathbb{Z} \\ k \neq \bar{n}}} \alpha_{k-\bar{n}} r_{k}-\frac{2 i \sqrt{2 \pi}}{1+\sqrt{\omega \bar{n}-i p}}
$$

Since the last term is analytic in a neighborhood of $\bar{p}$ and $\left\{t_{n}\right\},\left\{r_{n}\right\} \in \ell_{2}(\mathbb{Z} \backslash\{\bar{n}\})$ are both analytic, as it follows applying Lemma 3.1 above to (3.10) and (3.11), it is sufficient to prove that

$$
\sum_{\substack{k \in \mathbb{Z} \\ k \neq \bar{n}}} \alpha_{k-\bar{n}} \tilde{t}_{k} \neq 0
$$

where

$$
\left.\tilde{t}_{n} \equiv t_{n}(p)\right|_{p=\bar{p}}
$$

Assume that the contrary is true: from equation (3.10) we obtain

$$
\sum_{\substack{n \in \mathbb{Z} \\ n \neq \bar{n}}}\left(4 \pi \alpha_{0}+\sqrt{\omega n-i \bar{p}}\right)\left|\tilde{t}_{n}\right|^{2}=-4 \pi \sum_{\substack{n, k \in \mathbb{Z} \\ n, k \neq \bar{n}, n \neq k}} \tilde{t}_{n}^{*} \alpha_{k-n} \tilde{t}_{k}-4 \pi \sum_{\substack{n \in \mathbb{Z} \\ n \neq \bar{n}}} \alpha_{n-\bar{n}}^{*} \tilde{t}_{n}^{*}=
$$




$$
=-4 \pi \sum_{\substack{n, k \in \mathbb{Z} \\ n, k \neq \bar{n}, n \neq k}} \tilde{t}_{n}^{*} \alpha_{k-n} \tilde{t}_{k}
$$

where we have used condition 2 in (2.9). The previous equation implies (the right hand side is real) $\tilde{t}_{n}=0, \forall n<\bar{N}=\frac{i \bar{p}}{\omega}$ and then, since $-1<\bar{N}<0$, $\tilde{t}_{n}=0, \forall n<0$. Hence from (3.10) we have, $\forall n<0$,

$$
\sum_{\substack{k \geq 0 \\ k \neq \bar{n}}} \alpha_{k-n} \tilde{t}_{k}+\alpha_{\bar{n}-n}=0
$$

Now supposing without loss of generality that $\tilde{t}_{0} \neq 0$ and setting $T_{n}=\tilde{t}_{n-1}$, $n \neq \bar{n}+1$, and $T_{\bar{n}+1}=1$, we obtain, $\forall n \geq 0$,

$$
\sum_{k=1}^{\infty} \alpha_{k+n} T_{k}=0
$$

and using the genericity condition (3.3) (as in the proof of Lemma 3.1) we get $T_{1}=t_{0}=0$, which is a contradiction.

In conclusion $q_{\bar{n}}$ is analytic in a neighborhood of $\bar{p}$ : analyticity of $q_{n}, n \neq \bar{n}$ is then a straightforward consequence of analyticity of $\left\{r_{n}\right\},\left\{t_{n}\right\}$ and decomposition $q_{n}=r_{n}+t_{n} q_{\bar{n}}$. The proof is then completed, since $r_{n}$ and $t_{n}$ belong to $\ell_{2}(\mathbb{Z} \backslash\{\bar{n}\})$ in a neighborhood of $p=\bar{p}$.

\subsection{Behavior at $p=0$}

We shall now study the behavior of the solution of (3.5) on the imaginary axis at the origin. With the choice (2.15) for the branch cut of the square root, it is clear that we must expect branch points of $\tilde{q}(p)$, solution of (3.4), at $p=i \omega n$, $n \in \mathbb{Z}$, which should imply a branch point at $p=0$ for each $q_{n}$ in (3.5).

We are going to show that $q_{n}, n \in \mathbb{Z}$ has a branch point at $p=0$. The nonresonant case and the resonant one will be treated separately.

\section{Proposition 3.4 (non-resonant case)}

If $\left(4 \pi \alpha_{0}\right)^{2} \neq N \omega, \forall N \in \mathbb{N}$ and $\left\{\alpha_{n}\right\}$ satisfies 2.9) and 3.3) (genericity condition), the solution of equation (3.5) has the form $q_{n}(p)=c_{n}(p)+d_{n}(p) \sqrt{p}$, $n \in \mathbb{Z}$, in an imaginary neighborhood of $p=0$, where the functions $c_{n}(p)$ and $d_{n}(p)$ are analytic at $p=0$.

Proof: Setting $q_{n}=r_{n}+t_{n} q_{0}, n \neq 0$ and choosing a solution $\left\{t_{n}\right\} \in \ell_{2}(\mathbb{Z} \backslash$ $\{0\}$ ) of the system of equations (3.10) with $\bar{n}=0$, we obtain that $\left\{r_{n}\right\}$ must satisfy (3.11). It is easy to see that the result of Lemma 3.1 holds also in a neighborhood of $\bar{p}=0$ with $\bar{n}=0$, so that $\left\{r_{n}\right\},\left\{t_{n}\right\} \in \ell_{2}(\mathbb{Z} \backslash\{0\})$ are unique and analytic at $p=0$.

Thus it is sufficient to prove that $q_{0}$, which is solution of

$$
\left[4 \pi \alpha_{0}+\sqrt{-i p}+4 \pi \sum_{\substack{k \in \mathbb{Z} \\ k \neq 0}} \alpha_{k} t_{k}\right] q_{0}=-4 \pi \sum_{\substack{k \in \mathbb{Z} \\ k \neq 0}} \alpha_{k} r_{k}-\frac{2 i \sqrt{2 \pi}(1-\sqrt{-i p})}{1+i p}
$$


has the required behavior near $p=0$.

First, setting $t_{n}^{0}=t_{n}(p=0)$, we have to prove that

$$
\sum_{\substack{k \in \mathbb{Z} \\ k \neq 0}} \alpha_{k} t_{k}^{0} \neq-\alpha_{0}
$$

but, assuming that the contrary is true and multiplying both sides of equation (3.10) by $t_{n}^{*^{*}}$ and summing over $n \in \mathbb{Z}, n \neq 0$, one has

$$
\sum_{n \in \mathbb{Z}} \sqrt{\omega n}\left|t_{n}^{0}\right|^{2}=-4 \pi \sum_{\substack{n, k \in \mathbb{Z} \\ n, k \neq 0}} t_{n}^{0 *} \alpha_{k-n} t_{k}^{0}+4 \pi \alpha_{0}
$$

and then, because of genericity condition (3.3), $\left\{t_{n}^{0}\right\}=0, \forall n \in \mathbb{Z} \backslash\{0\}$, which is impossible, since $\left\{t_{n}\right\}$ solves (3.10).

Now, calling

$$
F \equiv 4 \pi \sum_{\substack{k \in \mathbb{Z} \\ k \neq 0}} \alpha_{k} t_{k}
$$

and

$$
G \equiv-4 \pi \sum_{\substack{k \in \mathbb{Z} \\ k \neq 0}} \alpha_{k} r_{k}
$$

we have

$$
\left[4 \pi \alpha_{0}+\sqrt{-i p}+F\right] q_{0}=G+\frac{2 i \sqrt{2 \pi}(1-\sqrt{-i p})}{1+i p}
$$

and

$$
q_{0}=F^{\prime}+\sqrt{p} G^{\prime}
$$

where $F^{\prime}$ is analytic in a neighborhood of $p=0$, because of analyticity of $F$ and $G$, and

$$
G^{\prime} \equiv-\frac{2 i \sqrt{-2 \pi i}\left(4 \pi \alpha_{0}+F+1\right)+\sqrt{-i}(1+i p) G}{(1+i p)\left[\left(4 \pi \alpha_{0}+F\right)^{2}+i p\right]}
$$

The resonant case, i.e. $4 \pi \alpha_{0}=-\sqrt{\omega N}$ for some $N \in \mathbb{N}$, is not so different from the non-resonant one and we shall prove that the solution has the same behavior at the origin. The proof is slightly different because we need to show the absence of a pole at $p=0$ : from (3.5) one has

$$
q_{N}(p)=\frac{4 \pi}{\sqrt{\omega N}-\sqrt{\omega N-i p}}\left\{\sum_{\substack{k \in \mathbb{Z} \\ k \neq 0}} \alpha_{k} q_{n+k}(p)+\frac{i}{\sqrt{2 \pi}} \frac{1-\sqrt{\omega N-i p}}{1+i p-\omega N}\right\}
$$

and the coefficients have a singularity at $p=0$.

We are going to prove that in fact the solution has no pole at the origin: proceeding as in the proof of Proposition 3.3 let us begin with a preliminary result, which take the place of Lemma 3.1 
Lemma 3.2 Let (2.9) and the genericity condition (3.3) be satisfied by $\left\{\alpha_{n}\right\}$. The system of equations

$$
r_{n}=\frac{4 \pi}{\sqrt{\omega N}-\sqrt{\omega n-i p}}\left\{\sum_{\substack{k \in \mathbb{Z} \\ k \neq 0,-n}} \alpha_{k} r_{n+k}+h_{n}(p)\right\}
$$

has a unique solution $\left\{r_{n}\right\} \in \ell_{2}(\mathbb{Z} \backslash\{N\})$ in a pure imaginary neighborhood of $p=0$, for every $h_{n}(p)$ such that

$$
h_{n}^{\prime}(p) \equiv \frac{h_{n}(p)}{\sqrt{\omega N}-\sqrt{\omega n-i p}}
$$

belongs to $\ell_{2}(\mathbb{Z} \backslash\{N\})$.

Moreover, if $h_{n}(p)$ is analytic in a neighborhood of $p=0$, the solution is analytic in the same neighborhood.

Proof: We shall proceed as in the proof of Proposition [3.3 separating the contribution of $r_{N}$, which may be singular: setting $r_{n}=u_{n}+v_{n} r_{N}, n \neq 0, N$, on (3.13), one has

$$
\begin{gathered}
u_{n}+v_{n} r_{N}=\frac{4 \pi}{\sqrt{\omega N}-\sqrt{\omega n-i p}}\left\{\alpha_{N-n} r_{N}+\sum_{\substack{k \in \mathbb{Z} \\
k \neq 0,-n, N-n}} \alpha_{k}\left(u_{n+k}+v_{n+k} r_{N}\right)\right\}+ \\
+\frac{2 i \sqrt{2 \pi}}{\sqrt{\omega N}-\sqrt{\omega n-i p}} \frac{1-\sqrt{\omega n-i p}}{1+i p-\omega n}
\end{gathered}
$$

and requiring that $\left\{v_{n}\right\}, n \neq 0, N$, solves

$$
v_{n}=\frac{4 \pi}{\sqrt{\omega N}-\sqrt{\omega n-i p}} \sum_{\substack{k \in \mathbb{Z} \\ k \neq 0,-n, N-n}} \alpha_{k} v_{n+k}+\frac{4 \pi \alpha_{N-n}}{\sqrt{\omega N}-\sqrt{\omega n-i p}}
$$

the equation for $\left\{u_{n}\right\}, n \neq 0, N$, becomes

$$
u_{n}=\frac{4 \pi}{\sqrt{\omega N}-\sqrt{\omega n-i p}}\left\{\sum_{\substack{k \in \mathbb{Z} \\ k \neq 0,-n, N-n}} \alpha_{k} u_{n+k}+\frac{i}{\sqrt{2 \pi}} \frac{1-\sqrt{\omega n-i p}}{1+i p-\omega n}\right\}
$$

Moreover $r_{N}$ satisfies

$$
r_{N}=\frac{4 \pi}{\sqrt{\omega N}-\sqrt{\omega N-i p}}\left\{\sum_{\substack{k \in \mathbb{Z} \\ k \neq 0,-N}} \alpha_{k}\left(u_{k}+v_{k} r_{N}\right)+\frac{i}{\sqrt{2 \pi}} \frac{1-\sqrt{\omega n-i p}}{1+i p-\omega n}\right\}
$$

or

$$
\begin{gathered}
{\left[\sqrt{\omega N}-\sqrt{\omega N-i p}-4 \pi \sum_{\substack{k \in \mathbb{Z} \\
k \neq 0, N}} \alpha_{k-N} v_{k}\right] r_{N}=} \\
=4 \pi \sum_{\substack{k \in \mathbb{Z} \\
k \neq 0, N}} \alpha_{k-N} u_{k}+\frac{i}{\sqrt{2 \pi}} \frac{1-\sqrt{\omega n-i p}}{1+i p-\omega n}
\end{gathered}
$$


Applying the discussion contained in the proof of Lemma 3.1 it is not difficult to see that the solutions of equations (3.15) and (3.14) are analytic in a neighborhood of the origin and belong to $\ell_{2}(\mathbb{Z} \backslash\{0, N\})$. Therefore it remains to prove that (setting $v_{n}^{0}=v_{n}(p=0)$ )

$$
\sum_{\substack{k \in \mathbb{Z} \\ k \neq 0, N}} \alpha_{k-N} v_{k}^{0} \neq 0
$$

but the argument in the proof of Proposition 3.3 excludes this possibility, if $\left\{\alpha_{n}\right\}$ satisfies the genericity condition. The proof is then completed, because analyticity of $r_{N}$ implies analyticity of all $r_{n}, n \neq 0, N$.

\section{Proposition 3.5 (resonant case)}

If $\left(4 \pi \alpha_{0}\right)^{2}=N \omega$, for some $N \in \mathbb{N}$ and $\left\{\alpha_{n}\right\}$ satisfies (2.9) and (3.3) (genericity condition), the solution of equation (3.5) has the form $q_{n}(p)=c_{n}(p)+d_{n}(p) \sqrt{p}$, $n \in \mathbb{Z}$, in an imaginary neighborhood of $p=0$, where the functions $c_{n}(p)$ and $d_{n}(p)$ are analytic at $p=0$.

Proof: See the proof of Proposition 3.4 and Lemma 3.2 above.

\subsection{Complete ionization in the generic case}

Summing up the results about the behavior of the Laplace transform $\tilde{q}(p)$ of $q(t)$ we can state the following

Theorem 3.1 If $\left\{\alpha_{n}\right\}$ satisfies (2.9) and the genericity condition (3.3) with respect to $\mathcal{T}$, as $t \rightarrow \infty$,

$$
|q(t)| \leq A t^{-\frac{3}{2}}+R(t)
$$

where $A \in \mathbb{R}$ and $R(t)$ has an exponential decay, $R(t) \sim C e^{-B t}$ for some $B>0$.

Proof: Propositions 3.23 .3 and 3.4 guarantee that $\tilde{q}(p)$ is analytic on the closed right half plane, except branch point singularities on the imaginary axis at $p=i \omega n, n \in \mathbb{Z}$.

Therefore we can chose a integration path for the inverse of Laplace transform of $\tilde{q}(q)$ along the imaginary axis like in [4.

Proposition 3.4 implies that the contribution of the branch point at $p=0$ is given by the integral

$$
2 i \int_{0}^{\infty} d p \sqrt{p} G^{\prime}(-p) e^{-p t}
$$

where $G^{\prime}$, defined in (3.12), is a bounded analytic function on the negative real line: from explicit expression of $F$ and $G$ and equations (3.11) and (3.10), it is clear that $G^{\prime}$ is analytic and $\lim _{p \rightarrow \infty} G^{\prime}(-p)=0$ on the real line. So that the corresponding asymptotic behavior as $t \rightarrow \infty$ is

$$
\left|\int_{0}^{\infty} d p \sqrt{p} G^{\prime}(-p) e^{-p t}\right| \leq C \int_{0}^{\infty} d p \sqrt{p} e^{-p t}=A t^{-\frac{3}{2}}
$$


Let us consider now the contribution of branch points at $p=i \omega n, n \neq 0$ : from Propositions 3.4 and 3.5 it follows that, in a neighborhood of $p=0$,

$$
q_{n}(p)=c_{n}(p)+d_{n}(p) \sqrt{p}
$$

where $c_{n}(p)$ and $d_{n}(p)$ are analytic at $p=0$. Moreover using the decomposition $q_{n}=r_{n}+t_{n} q_{0}, n \neq 0$, as in the proof of Proposition 3.4 and 3.5 and studying the equation (3.10) for $t_{n}$, we immediately obtain $\left\{d_{n}\right\} \in \ell_{1}(\mathbb{Z} \backslash\{0\})$, because of condition 2 in (2.9). Since $q_{n}(p)=\tilde{q}(p+i \omega n)$, the contribution of singularities at $p=i \omega n, n \neq 0$, is then given by

$$
\begin{aligned}
& 2 \sum_{\substack{n \in \mathbb{Z} \\
n \neq 0}} \int_{i \omega n-\infty}^{i \omega n} d p d_{n}(p-i \omega n) \sqrt{p-i \omega n} e^{p t}= \\
& =2 i \int_{0}^{\infty} d p\left\{\sum_{\substack{n \in \mathbb{Z} \\
n \neq 0}} d_{n}(-p) e^{i \omega n t}\right\} \sqrt{p} e^{-p t}=
\end{aligned}
$$

and the series

$$
\sum_{\substack{n \in \mathbb{Z} \\ n \neq 0}} d_{n}(-p) e^{i \omega n t}
$$

converges uniformly to a bounded function of $t$, because $\left\{d_{n}\right\} \in \ell_{1}(\mathbb{Z} \backslash\{0\})$.

Adding up the contributions of every branch cut, one obtain the required leading term in the asymptotic behavior. Indeed the rest function $R(t)$ is given by the contribution of poles outside the imaginary axis and then shows an exponential decay as $t \rightarrow \infty$.

A straightforward consequence of Theorem 3.1 is that the scalar product (and thus the survival probability of the bound state)

$$
\theta(t)=\left(\varphi_{\alpha(0)}, \Psi_{t}\right)_{L^{2}\left(\mathbb{R}^{3}\right)}
$$

tends to 0 when $t \rightarrow \infty$ :

Corollary 3.1 If $\left\{\alpha_{n}\right\}$ satisfies (2.9) and the genericity condition (3.3) with respect to $\mathcal{T}$, the system shows asymptotic complete ionization and, as $t \rightarrow \infty$,

$$
|\theta(t)| \leq D t^{-\frac{3}{2}}+E(t)
$$

where $D \in \mathbb{R}$ and $E(t)$ has an exponential decay.

Proof: The Laplace transform of $\theta(t)$ can be expressed in the following way (see the proof of Proposition 2.1)

$$
\tilde{\theta}(p)=\tilde{Z}_{1}(p)+\tilde{Z}_{2}(p) \tilde{q}(p)
$$

where $\tilde{Z}_{1}(p)$ is analytic on the closed right half plane and $\tilde{Z}_{2}(p)$ has only a branch point at the origin of the form $a_{1}+a_{2} \sqrt{p}$. 
Hence $\tilde{\theta}(p)$ has the same singularities as $\tilde{q}(p)$ and then its asymptotic behavior coincides with that of $q(t)$, i.e.

$$
|\theta(t)| \leq D t^{-\frac{3}{2}}+E(t)
$$

for some constant $D \in \mathbb{R}$ and for a bounded function $E(t)$ with exponential decay.

In the following we shall prove a stronger result about complete ionization of the system, namely that every state $\Psi \in L^{2}\left(\mathbb{R}^{3}\right)$ is a scattering state ${ }^{7}$ for the operator $H_{\alpha(t)}$, i.e.

$$
\lim _{t \rightarrow \infty} \frac{1}{t} \int_{0}^{t} d \tau\|F(|\vec{x}| \leq R) U(\tau, 0) \Psi\|^{2}=0
$$

where $F(S)$ is the multiplication operator by the characteristic function of the set $S \subset \mathbb{R}^{3}$ and $U(t, s)$ the unitary two-parameters family associated to $H_{\alpha(t)}$ (see (2.4)).

In order to prove (3.17), we first need to study the evolution of a generic initial datum in a suitable dense subset of $L^{2}\left(\mathbb{R}^{3}\right)$ and then we shall extend the result to every state using the unitarity of the evolution defined by (2.4) (see e.g. 9]).

Proposition 3.6 Let $\Psi \in C_{0}^{\infty}\left(\mathbb{R}^{3} \backslash\{0\}\right)$ a smooth function with compact support away from 0 and $q(t)$ be the solution of equation 2.6) with initial condition $\Psi_{0}=\Psi$. If $\left\{\alpha_{n}\right\}$ satisfies (2.9) and the genericity condition (3.3) with respect to $\mathcal{T}$, as $t \rightarrow \infty$,

$$
|q(t)| \leq A t^{-\frac{3}{2}}+R(t)
$$

where $A \in \mathbb{R}$ and $R(t)$ has an exponential decay, $R(t) \sim C e^{-B t}$ for some $B>0$.

Proof: The proof of Proposition 2.1] still applies, considering

$$
\theta^{\prime}(t) \equiv\left(\Psi, \Psi_{t}\right)_{L^{2}\left(\mathbb{R}^{3}\right)}
$$

instead of $\theta(t)$, so that $\tilde{q}(p)$, solution of (2.14) with initial condition $\Psi_{0}=\Psi$, is analytic $\forall p$ with $\Re(p)>0$.

Hence we can consider the Laplace transform of equation (2.6), which has the form (2.14) with

$$
f(p)=\sqrt{\frac{2}{\pi}} \sqrt{\frac{i}{p}} \int_{0}^{\infty} d t e^{-p t} \int_{\mathbb{R}^{3}} d^{3} \vec{k} \hat{\Psi}(\vec{k}) e^{-i k^{2} t}
$$

where $\hat{\Psi}(\vec{k})$ is the Fourier transform of $\Psi(\vec{x})$.

The equation for $\tilde{q}(p)$ is then given by

$$
\tilde{q}(p)=-\frac{4 \pi}{4 \pi \alpha_{0}+\sqrt{-i p}} \sum_{\substack{k \in \mathbb{Z} \\ k \neq 0}} \alpha_{k} \tilde{q}(p+i \omega k)+\frac{g(p)}{4 \pi \alpha_{0}+\sqrt{-i p}}
$$

\footnotetext{
${ }^{7}$ For the definition of scattering states of a time-dependent operator see e.g. 11$] 14$.
} 
where

$$
g(p)=\sqrt{\frac{2}{\pi}} \int_{0}^{\infty} d t e^{-p t} \int_{\mathbb{R}^{3}} d^{3} \vec{k} \hat{\Psi}(\vec{k}) e^{-i k^{2} t}
$$

It is now sufficient to show that the solution $\tilde{q}(p)$ is also analytic on the imaginary axis except at most square root branch points at $p=i \omega n$ as in the discussion of section 3.2 and 3.3 .

For every smooth function $\Psi$ with compact support, $\hat{\Psi}(\vec{k})$ is a smooth function with an exponential decay as $k \rightarrow \infty$, so that

$$
g(i s)=\lim _{r \rightarrow 0^{+}} \sqrt{\frac{2}{\pi}} \int_{\mathbb{R}^{3}} d^{3} \vec{k} \frac{\hat{\Psi}(\vec{k})}{r+\left(s+k^{2}\right) i}=-i \sqrt{\frac{2}{\pi}} \int_{\mathbb{R}^{3}} d^{3} \vec{k} \frac{\hat{\Psi}(\vec{k})}{s+k^{2}}
$$

is a bounded function for $s>0$. Hence the function $g(p)$ has no pole for $\Im(p) \in(0, \omega)$ and therefore the result contained in Proposition 3.3 still holds. Moreover

$$
g(0)=\sqrt{\frac{2}{\pi}} \int_{\mathbb{R}^{3}} d^{3} \vec{k} \hat{\Psi}(\vec{k}) \int_{0}^{\infty} d t e^{-i k^{2} t}=-i \sqrt{\frac{2}{\pi}} \int_{\mathbb{R}^{3}} d^{3} \vec{k} \frac{\hat{\Psi}(\vec{k})}{k^{2}}
$$

which is again bounded, so that $g(p)$ has at the origin at most a branch point singularity of the form $a(p)+b(p) \sqrt{p}$ : following the proofs of Proposition 3.4 and 3.5 we can show that $\tilde{q}(p)$ has the same behavior at the origin.

In conclusion the solution is analytic on the closed right half plane except branch points at $p=i \omega n, n \in \mathbb{Z}$, of the form $a(p)+b(p) \sqrt{p-i \omega n}$. The proof of Theorem 3.1 then implies that $q(t)$ has the prescribed behavior as $t \rightarrow \infty$.

Theorem 3.2 If $\left\{\alpha_{n}\right\}$ satisfies (2.9) and the genericity condition (3.3) with respect to $\mathcal{T}$, every $\Psi \in L^{2}\left(\mathbb{R}^{3}\right)$ is a scattering state of $H_{\alpha(t)}$, i.e.

$$
\lim _{t \rightarrow \infty} \frac{1}{t} \int_{0}^{t} d \tau\|F(|\vec{x}| \leq R) U(\tau, 0) \Psi\|^{2}=0
$$

Proof: We shall restrict the proof to the dense subset of $L^{2}\left(\mathbb{R}^{3}\right)$ given by smooth functions with compact support and then we shall extend the result to every state using the unitarity of the evolution defined by (2.5) (see e.g. 9]). Actually we are going to prove an equivalent but slightly different statement, i.e. $\forall \varepsilon>0$, there exists $t_{0}$ such that $\forall t>t_{0}$,

$$
\|F(|\vec{x}| \leq R) U(t, 0) \Psi\| \leq \varepsilon
$$

The evolution of an initial state $\Psi$ according to (2.5) is given by

$$
\Psi_{t}(\vec{x})=U(t, s) \Psi_{s}(\vec{x})=U_{0}(t-s) \Psi_{s}(\vec{x})+i \int_{s}^{t} d \tau q(\tau) U_{0}(t-\tau ; \vec{x})
$$

Moreover, since $\Psi_{t} \in \mathcal{D}\left(H_{\alpha(t)}\right)$, the following decomposition holds

$$
\Psi_{t}(\vec{x})=\varphi_{t}(\vec{x})+\frac{q(t)}{4 \pi|\vec{x}|}
$$


where $q(t)$ is the solution of $(\underline{2.6}), \varphi_{t} \in H_{\mathrm{loc}}^{2}\left(\mathbb{R}^{3}\right)$ and

$$
\varphi_{t}(0)=\alpha(t) q(t)
$$

We are going to show that, if $q(t) \in L^{1}\left(\mathbb{R}^{+}\right), \Psi_{t}$ satisfies the required property. Let us start analyzing the second term in (3.19): imposing the unitarity condition of the evolution we have

$$
\left\|\Psi_{s}\right\|^{2}=\left\|\Psi_{t}\right\|^{2}=\left\|U_{0}(t-s) \Psi_{s}(\vec{x})+i \int_{s}^{t} d \tau q(\tau) U_{0}(t-\tau ; \vec{x})\right\|^{2}
$$

and then

$$
\begin{gathered}
\left\|\int_{s}^{t} d \tau q(\tau) U_{0}(t-\tau ; \vec{x})\right\|^{2}=2 \Im\left(\int_{s}^{t} d \tau q(\tau) U_{0}(t-\tau ; \vec{x}), U_{0}(t-s) \Psi_{s}(\vec{x})\right)= \\
=2 \Im\left[\int_{s}^{t} d \tau q^{*}(\tau)\left(e^{-i H_{0}(\tau-s)} \Psi_{s}\right)(0)\right]
\end{gathered}
$$

but, using the decomposition (3.20),

$$
\begin{aligned}
\left(e^{-i H_{0}(s-\tau)} \Psi_{s}\right)(0) & =\left(e^{-i H_{0}(s-\tau)} \varphi_{s}\right)(0)+\int_{\mathbb{R}^{3}} d^{3} \vec{k} e^{-i k^{2}(\tau-s)} \frac{q(s)}{(2 \pi)^{3} k^{2}}= \\
= & \left(e^{-i H_{0}(s-\tau)} \varphi_{s}\right)(0)+\frac{q(s)}{4 \pi \sqrt{\pi i} \sqrt{\tau-s}}
\end{aligned}
$$

Since $\varphi_{s} \in H_{\mathrm{loc}}^{2}\left(\mathbb{R}^{3}\right)$, the absolute value of the first term on the right hand side is bounded by a constant $c(\tau, s)<\infty$ such that $c(s, s)=q(s)$ and

$$
\lim _{\tau \rightarrow \infty} c(\tau, s)=0
$$

Hence there exists $s_{1}(\varepsilon)>0$ such that, $\forall s>s_{1}$,

$$
2\left|\int_{s}^{t} d \tau q^{*}(\tau)\left(e^{-i H_{0}(s-\tau)} \varphi_{s}\right)(0)\right| \leq \frac{2 \varepsilon^{2}}{9}
$$

if $q(t) \in L^{1}\left(\mathbb{R}^{+}\right)$. Moreover by the same reason there exists $s_{2}(\varepsilon)>0$ such that $\forall s>s_{2}$,

$$
2\left|\int_{s}^{t} d \tau q^{*}(\tau) \frac{q(s)}{4 \pi \sqrt{\pi i} \sqrt{\tau-s}}\right| \leq \frac{2 \varepsilon^{2}}{9}
$$

Setting $s_{0}(\varepsilon)=\max \left(s_{1}(\varepsilon), s_{2}(\varepsilon)\right)$, one has $\forall s>s_{0}$

$$
\left\|\int_{s}^{t} d \tau q(\tau) U_{0}(t-\tau ; \vec{x})\right\| \leq \frac{2 \varepsilon}{3}
$$

so that the whole $L^{2}$-norm of the second term in decomposition (3.19) is suitably small for $s>s_{0}$.

On the other hand the first term in (3.19) is the free evolution of a $L^{2}$-function and hence there exists $\delta(\varepsilon)>0$ such that $\forall t>s+\delta$ and $\forall R<\infty$,

$$
\left\|F(|\vec{x}| \leq R) U(t-s) \Psi_{s}\right\| \leq \frac{\varepsilon}{3}
$$


Setting $t_{0}(\varepsilon)=s_{0}(\varepsilon)+\delta(\varepsilon)$, from (3.19), (3.21) and (3.22) one has

$$
\left\|F(|\vec{x}| \leq R) \Psi_{t}\right\| \leq \varepsilon
$$

$\forall t>t_{0}$, if $q(t) \in L^{1}\left(\mathbb{R}^{+}\right)$.

By Proposition 3.6 the inequality is then satisfied by every $\Psi \in C_{0}^{\infty}\left(\mathbb{R}^{3} \backslash\{0\}\right)$ : unitarity of the family $U(t, s)$ allows to extend the result to the whole Hilbert space $L^{2}\left(\mathbb{R}^{3}\right)$.

Corollary 3.2 If $\left\{\alpha_{n}\right\}$ satisfies (2.9) and the genericity condition with respect to $\mathcal{T}$ (3.3), the discrete spectrum of the Floquet operator associated to $H_{\alpha(t)}$,

$$
K \equiv-i \frac{\partial}{\partial t}+H_{\alpha(t)}
$$

is empty.

Proof: The result is a straightforward consequence of Theorem 3.2 every eigenvector of $K$ differs from a periodic function by a phase factor and hence can not satisfy (3.17).

\section{CASE II: $\alpha_{0}=0$}

If $\alpha(t)=\alpha_{0}=0$ does not depend on time, the problem has a simple solution: the spectrum of $H_{\alpha(t)}$ is absolutely continuous and equal to the positive real line, with a resonance at the origin; hence there is no bound state and the system shows complete ionization independently on the initial datum.

On the other hand if $\alpha(t)$ is a zero mean function, we shall see that the genericity condition (3.3) is still needed to have complete ionization.

So let us assume that $\alpha_{0}=0$, the normalization (3.1) holds and the initial datum is given by (2.7): equation (2.14) then becomes

$$
\tilde{q}(p)=-4 \pi \sqrt{\frac{i}{p}} \sum_{\substack{k \in \mathbb{Z} \\ k \neq 0}} \alpha_{k} \tilde{q}(p+i \omega k)-2 i \sqrt{\frac{2 \pi i}{p}} \frac{1-\sqrt{-i p}}{1+i p}
$$

with the choice (2.15) for the branch cut of $\sqrt{p}$. By Proposition 2.1 the solution is analytic on the open right half plane. In the following section we shall study the singularities on the imaginary axis.

\subsection{Singularities on the imaginary axis}

Setting $q_{n}(p) \equiv \tilde{q}(p+i \omega n), p \in \mathcal{I}=[0, \omega)$, as in Section 3.1, equation (4.1) assumes the form (3.5),

$$
q(p)=\mathcal{M}(p) q(p)+o(p)
$$


with

$$
(\mathcal{M} q)_{n}(p) \equiv-\frac{4 \pi}{\sqrt{\omega n-i p}} \sum_{\substack{k \in \mathbb{Z} \\ k \neq 0}} \alpha_{k} q_{n+k}(p)
$$

and $o(p)=\left\{o_{n}(p)\right\}_{n \in \mathbb{Z}}$,

$$
o_{n}(p) \equiv-\frac{2 i \sqrt{2 \pi}}{\sqrt{\omega n-i p}(1+\sqrt{\omega n-i p})}
$$

Proposition 4.1 For $p \in \mathcal{I}, \Re(p)=0, p \neq 0, \mathcal{M}(p)$ is an analytic operatorvalued function and $\mathcal{M}(p)$ is a compact operator on $\ell_{2}(\mathbb{Z})$.

Proof: See the proof of Proposition 3.1

Proposition 4.2 There exists a unique solution $q_{n}(p) \in \ell_{2}(\mathbb{Z})$ of 4.2 ) and it is analytic on the imaginary axis for $p \neq 0$.

Proof: See the proof of Proposition 3.2

Proposition 4.3 If $\left\{\alpha_{n}\right\}$ satisfies (2.9) and the genericity condition (3.3), the solution of equation (4.2) has the form $q_{n}(p)=c_{n}(p)+d_{n}(p) \sqrt{p}, n \in \mathbb{Z}$, in a neighborhood of $p=0$, where the functions $c_{n}(p)$ and $d_{n}(p)$ are analytic at $p=0$.

Proof: Let us proceed as in the proof of Proposition 3.4 setting $q_{n}=$ $r_{n}+t_{n} q_{0}, n \in \mathbb{Z} \backslash\{0\}$, where $\left\{t_{n}\right\}$ is the solution of

$$
t_{n}=-\frac{4 \pi}{\sqrt{\omega n-i p}} \sum_{\substack{k \in \mathbb{Z} \\ k \neq 0,-n}} \alpha_{k} t_{n+k}-\frac{4 \pi \alpha_{-n}}{\sqrt{\omega n-i p}}
$$

A slightly different version of Lemma 3.1 guarantees that the solution $\left\{t_{n}\right\} \in$ $\ell_{2}(\mathbb{Z} \backslash\{0\})$ is unique and analytic at $p=0$.

By means of this substitution we obtain

$$
r_{n}=-\frac{4 \pi}{\sqrt{\omega n-i p}} \sum_{\substack{k \in \mathbb{Z} \\ k \neq 0,-n}} \alpha_{k} r_{n+k}-\frac{2 i \sqrt{2 \pi}}{\sqrt{\omega n-i p}(1+\sqrt{\omega n-i p})}
$$

and

$$
q_{0}=-\frac{4 \pi}{\sqrt{-i p}} \sum_{\substack{k \in \mathbb{Z} \\ k \neq 0}} \alpha_{k}\left(r_{k}+t_{k} q_{0}\right)-\frac{2 i \sqrt{2 \pi}}{\sqrt{-i p}(1+\sqrt{-i p})}
$$

or

$$
(\sqrt{-i p}+F) q_{0}=G-\frac{2 \sqrt{2 \pi}}{1+\sqrt{-i p}}
$$


where (like in the proof of Proposition 3.4)

$$
F \equiv 4 \pi \sum_{\substack{k \in \mathbb{Z} \\ k \neq 0}} \alpha_{k} t_{k}
$$

and

$$
G \equiv-4 \pi \sum_{\substack{k \in \mathbb{Z} \\ k \neq 0}} \alpha_{k} r_{k}
$$

Moreover $F(0) \neq 0$, because of genericity condition (3.3) (see the proof of Proposition 3.4), $F$ and $G$ are analytic in a neighborhood of $p=0$ (see Lemma 3.1), so that

$$
q_{0}=F^{\prime}+\sqrt{p} G^{\prime}
$$

where $F^{\prime}$ and $G^{\prime}$ are analytic and

$$
G^{\prime} \equiv \frac{2 \sqrt{-2 \pi i}(F+1)-\sqrt{-i}(1+i p) G}{(1+i p)\left(F^{2}+i p\right)}
$$

\subsection{Complete ionization in the generic case}

As in section 3 we can now state the main result:

Theorem 4.1 If $\left\{\alpha_{n}\right\}$ satisfies (2.9) and the genericity condition (3.3) with respect to $\mathcal{T}$, as $t \rightarrow \infty$,

$$
|q(t)| \leq A t^{-\frac{3}{2}}+R(t)
$$

where $A \in \mathbb{R}$ and $R(t)$ has an exponential decay, $R(t) \sim C e^{-B t}$ for some $B>0$.

Proof: See the proof of Theorem 3.1

Corollary 4.1 If $\left\{\alpha_{n}\right\}$ satisfies (2.9) and the genericity condition (3.3) with respect to $\mathcal{T}$, the system shows asymptotic complete ionization and, as $t \rightarrow \infty$,

$$
|\theta(t)| \leq D t^{-\frac{3}{2}}+E(t)
$$

where $D \in \mathbb{R}$ and $E(t)$ has an exponential decay.

Proof: See the proof of Corollary 3.1

Theorem 4.2 If $\left\{\alpha_{n}\right\}$ satisfies (2.9) and the genericity condition (3.9) with respect to $\mathcal{T}$, every $\Psi \in L^{2}\left(\mathbb{R}^{3}\right)$ is a scattering state of $H_{\alpha(t)}$, i.e.

$$
\lim _{t \rightarrow \infty} \frac{1}{t} \int_{0}^{t} d \tau\|F(|\vec{x}| \leq R) U(\tau, 0) \Psi\|^{2}=0
$$

Moreover the discrete spectrum of the Floquet operator is empty.

Proof: See the proof of Proposition 3.6 and Theorem 3.2 


\section{CASE III: $\alpha_{0}>0$}

To complete the analysis of the problem, we shall consider the case of mean greater than 0: taking the normalization (3.1) and the initial condition (2.7), (2.14) assumes the form (3.4):

$$
\tilde{q}(p)=-\frac{4 \pi}{4 \pi \alpha_{0}+\sqrt{-i p}} \sum_{\substack{k \in \mathbb{Z} \\ k \neq 0}} \alpha_{k} \tilde{q}(p+i \omega k)-\frac{2 i \sqrt{2 \pi}}{4 \pi \alpha_{0}+\sqrt{-i p}} \frac{1-\sqrt{-i p}}{1+i p}
$$

Analyticity of the solution on the open right half plane is a consequence of Proposition 2.1

Moreover, following the discussion contained in section 3 and setting $q_{n}(p) \equiv$ $\tilde{q}(p+i \omega n), \Im(p) \in[0, \omega)$, the equation assumes the form (3.5]).

Let us now consider the behavior on the imaginary axis: singularities for $\Re(p)=$ 0 are associated to zeros of $4 \pi \alpha_{0}+\sqrt{\omega n+s}, s \in[0, \omega)$, but, since $\alpha_{0}>0$, it is clear that the expression can not have zeros on the imaginary axis. Hence the proof of Proposition 3.2 can be extended to the closed right half plane except the origin:

Proposition 5.1 If $\left\{\alpha_{n}\right\}$ satisfies (2.9), the solution $\tilde{q}(p)$ of (5.1) is unique and analytic for $\Re(p) \geq 0, p \neq i \omega n, n \in \mathbb{Z}$.

Proof: See the proof of Proposition 3.2 Propositions 3.1 and 2.2 and the previous discussion.

Moreover the behavior at the origin is described by the following

Proposition 5.2 If $\left\{\alpha_{n}\right\}$ satisfies (2.9) and the genericity condition with respect to $\mathcal{T}$ (3.3), then, in an imaginary neighborhood of $p=i \omega n, n \in \mathbb{Z}$, the solution of equation (5.1) has the form $\tilde{q}(p)=c_{n}(p)+d_{n}(p) \sqrt{p-i \omega n}$, where the functions $c_{n}(p)$ and $d_{n}(p)$ are analytic at $p=i \omega n$.

Proof: The proof of Proposition 3.4 still applies with only one difference: since, independently on $\omega$, the solution can not have a pole on the imaginary axis, we need not to distinguish between the resonant case and the non-resonant one.

We can now prove asymptotic complete ionization of the system:

Theorem 5.1 If $\left\{\alpha_{n}\right\}$ satisfies (2.9) and the genericity condition 3.3) with respect to $\mathcal{T}$, as $t \rightarrow \infty$,

$$
|q(t)| \leq A t^{-\frac{3}{2}}+R(t)
$$

where $A \in \mathbb{R}$ and $R(t)$ has an exponential decay, $R(t) \sim C e^{-B t}$ for some $B>0$. Moreover the system shows asymptotic complete ionization and, as $t \rightarrow \infty$,

$$
|\theta(t)| \leq D t^{-\frac{3}{2}}+E(t)
$$

where $D \in \mathbb{R}$ and $E(t)$ has an exponential decay. 
Proof: See the proof of Theorem 3.1 and Corollary 3.1

Theorem 5.2 If $\left\{\alpha_{n}\right\}$ satisfies (2.9) and the genericity condition (3.9) with respect to $\mathcal{T}$, every $\Psi \in L^{2}\left(\mathbb{R}^{3}\right)$ is a scattering state of $H_{\alpha(t)}$, i.e.

$$
\lim _{t \rightarrow \infty} \frac{1}{t} \int_{0}^{t} d \tau\|F(|\vec{x}| \leq R) U(\tau, 0) \Psi\|^{2}=0
$$

Moreover the discrete spectrum of the Floquet operator is empty.

Proof: See the proof of Proposition 3.6 and Theorem 3.2

Remark: If $\alpha(t) \geq 0, \forall t \in \mathbb{R}^{+}$, Proposition 5.2 holds without the genericity condition on the Fourier coefficients of $\alpha(t)$ : for instance the genericity condition enters (see the proof of Proposition [3.4) in the proof of absence of non-zero solutions of the homogeneous equation

$$
t_{n}=-\frac{4 \pi}{4 \pi \alpha_{0}+\sqrt{\omega n+s}} \sum_{\substack{k \in \mathbb{Z} \\ k \neq 0,-n}} \alpha_{k} t_{n+k}
$$

where $s \in[0, \omega)$. Let us suppose that there exists a non-zero solution $\left\{T_{n}\right\} \in$ $\ell_{2}(\mathbb{Z})$. Multiplying both sides of the equation by $T_{n}^{*}$, one has

$$
\sum_{\substack{n \in \mathbb{Z} \\ n \neq 0}} \sqrt{\omega n+s}\left|T_{n}\right|^{2}=-4 \pi \sum_{\substack{n, k \in \mathbb{Z} \\ n, k \neq 0}} T_{n}^{*} \alpha_{k-n} T_{k}
$$

Since the right hand side is real, $T_{n}=0, \forall n<0$. Moreover, fixing $T_{0}=0$ and setting

$$
T(t) \equiv \sum_{n \in \mathbb{Z}} T_{n} e^{-i \omega n t}
$$

it follows that

$$
-4 \pi \sum_{n, k \in \mathbb{Z}} T_{n}^{*} \alpha_{k-n} T_{k}=-4 \pi(T(t), \alpha(t) T(t))_{L^{2}([0, T])} \leq 0
$$

because $\alpha(t) \geq 0, \forall t \in[0, T]$, but the left hand side is positive and then $Q_{n}=0$, $\forall n \in \mathbb{Z}$.

\section{Conclusions and Perspectives}

In sections 3,4 and 5 we have proved that, under the genericity condition on $\alpha(t)$, the system defined in section 2 shows asymptotic complete ionization, independently on its frequency.

If $\inf (\alpha(t))<0$, the genericity condition may be a necessary condition to have complete ionization: for example, in one dimension, it is possible to exhibit 
(see 4) explicit functions $\alpha(t)$ for which the genericity condition fails ${ }^{8}$ and the ionization is not complete. On the other hand, also in one dimension, it is not known whether the condition is necessary. It would be interesting to check if non generic $\alpha(t)$ gives rise to asymptotic partial ionization in three dimensions. A possible way to investigate this problem is the analysis of the discrete spectrum of the Floquet operator. If one can find an explicit relation between existence of eigenvalues of the Floquet operator and the genericity condition, it would be probably easy to check if the condition is truly necessary.

On the other hand, as we expected, if $\alpha(t)$ is positive at any time, no further condition on $\alpha(t)$ is required to prove complete ionization.

Two interesting future applications of these methods can be the problem of complete ionization for moving point interactions and for $N$ time-dependent point interactions. Indeed there are simple examples in which asymptotic complete ionization occurs also for moving sources (see $\underline{3}$ ).

Acknowledgments: M.C. is very grateful to Prof. Ludwik Dabrowski and the INTAS Research Project nr. 00-257 of European Community, "Spectral Problems for Schrödinger-Type Operators", for the support.

\footnotetext{
${ }^{8} \mathrm{~A}$ simple example of $\alpha(t)$, for which the genericity condition is not satisfied is the geometric series, $\alpha_{n}=\lambda^{|n|}$ for some $\lambda<1$.
} 


\section{References}

[1] S.A. Albeverio, F. Gesztesy, R. Hoegh-Krohn, H. Holden, Solvable Models in Quantum Mechanics, Springer-Verlag, New York, 1988.

[2] F.A. Berezin, L.D. Faddeev, A Remark on Schrödinger Equation with a Singular Potential, Sov. Math. Dokl. 2, 372-375, 1961.

[3] M. Correggi, G.F. Dell'Antonio, Rotating Singular Perturbations of the Laplacian, preprint math-ph/0307056, to appear in Ann. H. Poincaré, 2004.

[4] O. Costin, R.D. Costin, J.L. Lebowitz, A. Rokhlenko, Evolution of a Model Quantum System under Time Periodic Forcing: Conditions for Complete Ionization, Comm. Math. Phys. 221, no.1 1-26, 2001.

[5] O. Costin, J.L. Lebowitz, A. Rokhlenko, Decay versus Survival of a Localized State Subjected to Harmonic Forcing: Exact Results, J. Phys. A: Math. Gen. 35, 8943-8951, 2002.

[6] O. Costin, J.L. Lebowitz, A. Rokhlenko, Exact Results for the Ionization of a Model Quantum System, J. Phys. A: Math. Gen. 33, 6311-6319, 2000.

[7] O. Costin, R.D. Costin, J.L. Lebowitz, Transition to the Continuum of a Particle in Time-Periodic Potentials, in Advances in Differential Equations and Mathematical Physics, Birmingham, AL, 2002, Contemp. Math. 327, 75-86, AMS, Providence, 2003.

[8] O. Costin, J.L. Lebowitz, A. Rokhlenko, Ionization of a Model Atom: Exact Results and Connection with Experiment, preprint physics/9905038, 1999.

[9] G.F. Dell'Antonio, R. Figari, A. Teta, Schrödinger Equation with Moving Point Interactions in Three Dimensions, in Stochastic Processes, Physics and Geometry: New Interplays, Leipzig, 1999, CMS Conference Proceedings 28, 99-113, AMS, Providence, 2000.

[10] G.F. Dell'Antonio, Point Interactions, in Mathematical Physics in Mathematics and Physics, Siena, 2000, Fields Institute Communications 30, 139-150, AMS, Providence, 2001.

[11] V. Enss, K. Veselic, Bound States and Propagating States for Time-dependent Hamiltonians, Ann. Inst. H. Poincaré A 39, 159-191, 1983.

[12] R. Figari, Time Dependent and Non Linear Point Interactions, in Proceedings of Mathematical Physics and Stochastic Analysis, Lisbon, 1998, 184-197, World Scientific Publisher, New York, 2000.

[13] S. Graffi, V. Grecchi, H.J. Silverstone, Resonances and Convergence of Perturbative Theory for N-body Atomic Systems in External AC-electric Field, Ann. Inst. H. Poincaré A, 42, 215-234, 1985. 
[14] J.S. Howland, Stationary Scattering Theory for Time-dependent Hamiltonians, Math. Ann. 207, 315-335, 1974.

[15] J.S. Howland, Scattering Theory for Hamiltonians Periodic in Time, Indiana Univ. Math. J. 28 No.3, 471-494, 1979.

[16] D. Porter, D.S.G. Stirling, Integral Equations, Cambridge University Press, Cambridge, 1990.

[17] M. Reed, B. Simon, Methods of Modern Mathematical Physics, Vol.I: Functional Analysis, Academic Press, San Diego, 1975.

[18] M.R. SAyapova, D.R. Yafaev, The Evolution Operator for Timedependent Potentials of Zero Radius, Proc. Stek. Inst. Math. 2, 173$180,1984$.

[19] D.R. Yafaev, Scattering Theory for Time-dependent Zero-range Potentials, Ann. Inst. H. Poincaré A 40, 343-359, 1984.

[20] K. Yajima, H. Kitada, Bound States and Scattering States for Time Periodic Hamiltonians, Ann. Inst. H. Poincaré A 39, 145-157, 1983. 